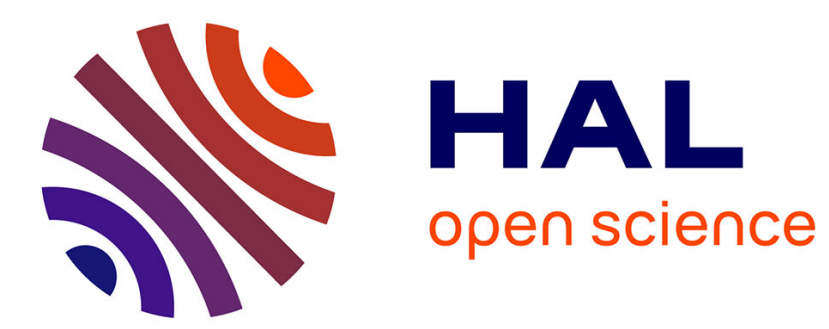

\title{
DNA Content, Cell Size, and Cell Senescence
}

Reiner A. Veitia

\section{To cite this version:}

Reiner A. Veitia. DNA Content, Cell Size, and Cell Senescence. Trends in Biochemical Sciences, 2019, 44, pp.645 - 647. 10.1016/j.tibs.2019.04.013 . hal-03487958

\section{HAL Id: hal-03487958 \\ https://hal.science/hal-03487958}

Submitted on 20 Dec 2021

HAL is a multi-disciplinary open access archive for the deposit and dissemination of scientific research documents, whether they are published or not. The documents may come from teaching and research institutions in France or abroad, or from public or private research centers.
L'archive ouverte pluridisciplinaire HAL, est destinée au dépôt et à la diffusion de documents scientifiques de niveau recherche, publiés ou non, émanant des établissements d'enseignement et de recherche français ou étrangers, des laboratoires publics ou privés.

\section{다)(1) $(5$}

Distributed under a Creative Commons Attribution - NonCommercial| 4.0 International 
DNA content, cell size, and cell senescence.

Reiner A. Veitia ${ }^{1,2}$

${ }^{1}$ Institut Jacques Monod, Université Paris Diderot, Paris, France.

${ }^{2}$ Université Paris-Diderot, Paris, France.

Correspondence to Prof. Reiner A. Veitia

Institut Jacques Monod. 15 rue Hélène Brion, 75013 Paris. France

Phone: 33603516746

Email : reiner.veitia@ijm.fr 


\section{Abstract}

A recent study assessed the impact of cell size on various cell properties. Over-sized yeast cells display slow cell division, cytoplasmic dilution and transcriptomic and proteomic alterations. It highlights commonalities between aging yeast and mammalian cells, suggesting the existence of a range of DNA content:cell volume ratio ensuring optimal function. 
Cell size is a characteristic feature for any given cell type. Senescence in yeast and human cells is associated with an increase in cell size $([1,2]$ and references therein), which affects intracellular transport, surface-to-volume, and DNA content-to-nucleus/cytoplasm volume ratios. Chemical or genetic manipulations blocking cell-cycle progression can induce an artificial increase in cell size [3]. In a recent paper, Neurohr et al. used two different temperature sensitive alleles of $C d c 28$ to reversibly arrest budding yeast cells in $\mathrm{G} 1$ in order to study the impact of cell size on various cell properties [4]. For instance, mutants grown for 6 hours at the restrictive temperature undergo a cell volume increase from $65 \mathrm{fL}$ to up to $800 \mathrm{fL}$.

To disentangle the consequences of growing large from those of prolonged cell-cycle arrest, Neurohr et al. compared two cell populations: arrested over-sized cells and arrested cells prevented from growing large either by using the translation inhibitor cycloheximide or by limiting glucose. The former resumed proliferation more slowly than small cells. They also progressed more slowly into $S$ phase, which was paralleled by a delayed DNA replication. Indeed, all cell-cycle stages analyzed were delayed in cells that grew very large $(600 \mathrm{fL})$. Similar results were obtained with cells mutated for the formin Bni1, arrested in $\mathrm{G} 1$ with the pheromone a-factor. Further experiments showed that cell-cycle checkpoint activation contributes to the observed delays but other defects also impair cell-cycle progression [4].

A potential explanation for the cell-cycle delay might be the existence of limiting amounts of regulators in large cells. Consistently, attenuation of gene induction in large cells was shown for two cyclins. However, the impact of cell size on transcription induction goes well beyond these two cases. For instance, induction of GAL1 gene and of pheromone-induced genes was less efficient in large cells but not in those kept small with cycloheximide. Chromatin immunoprecipitation experiments showed that the repressor Gal80 was not removed from galactoseresponsive promoters, impairing recruitment of the TATA-binding protein and the RNA polymerase notably to the GAL1 promoter. Besides, defective pheromone MAPK signaling was found in large cells [4].

Consistent with previous results [5], cell growth rate (fL/h) was proportional to cell volume up to about $200 \mathrm{fL}$, after which a plateau started to appear [4]. In the first phase, a transcriptomic analysis showed that mRNAs levels increased proportionally 
with cell volume [4]. In the 'deceleration' phase, the proportionality with volume of most transcripts disappeared. In a particular condition, cytoplasmic and nuclear volumes increased by $9 x$ whereas protein content increased by only $5 x$. This trend was confirmed for several highly expressed proteins fused to fluorescent tags [4]. Consistently, quantitative mass spectrometry showed that total protein concentration decreased in large cells [4]. Specifically, protein content scaled with cell size up to 3 hours of G1 arrest but not afterwards. In short, lack of proportionality between cell volume and RNA/protein appears when cell volume exceeds $200 \mathrm{fL}$. In such conditions, the transcriptional and translational capacity of the cell becomes limiting, which results in the dilution of cytoplasmic components and in a decreased cell density.

A potential explanation for such effects could be that DNA itself becomes 'limiting' for sustaining transcription (and subsequent translation) in large cells. Moreover, cells arrested for more than 3 hours seem to trigger a 'stress response' characterized by the repression of genes encoding ribosome biogenesis factors and general transcription factors (TFs) [6]. This phenomenon affects both highly and poorly expressed genes. In addition to this stress response, as shown in Figure 1A$\mathrm{B}$, mere nucleo-cytoplasmic dilution is predicted to decrease transcription (and hence, translation) of both poorly and highly expressed genes, depending on promoter characteristics.

The authors also analyzed yeast strains with different ploidy levels (which correlate with cell size) and found that maximal growth rate of arrested cells correlated with ploidy level (triploid>diploid>haploid) [4]. To exclude that the observed differences were a consequence of differences in initial cell size, they examined cell growth of synchronized cdc28-mutants also lacking Mad1 and Bub2, which in the absence/presence of nocodazole produce haploid/diploid cells of almost identical cell sizes. As expected, diploid cells grew faster than haploids, suggesting that DNA content determines maximal growth rate. In line with previously discussed findings, GAL1 promoter induction and pheromone signaling was also more efficient in diploids than haploids of similar sizes. This suggests that an optimal range DNAcontent-to-nucleo/cytoplasm volume ratio is required to warrant an appropriate RNA and protein synthesis able of ensuring proper gene expression, cell proliferation and signaling. 
Although not directly addressed in the paper, nucleo-cytoplasmic dilution must also affect the concentration of macromolecular complexes. Consider the example of a complex $A_{a} B_{b} \ldots Z_{z}$. Let us assume that the multimer is dynamic and only its subunits are directly degraded [7]. The interaction of the latter is represented by a constant $K$. In these conditions, the steady state (ss) concentration of $A_{a} B_{b} \ldots Z_{z}$ is:

$$
[A a B b \ldots Z z]=K\left[A_{s s}\right]^{a}\left[B_{s s}\right]^{b} \ldots\left[Z_{s s}\right]^{z}
$$

Inspection of this equation shows that the (maximum) fold-reduction of $A_{a} B_{b} \ldots Z_{z}$ concentration is proportional to the power $a+b+\ldots+z$ of the dilution factor. This nonlinear dependence suggests that bigger complexes are more sensitive to nucleocytoplasmic dilution. To complicate matters, we can consider the case of enzymes with opposing activities (i.e., a protein kinase and a phosphatase) involved in signal transduction, which can be monomeric or multimeric. It is obvious that any imbalance between the opposed enzymatic activities, upon nucleo-cytoplasmic dilution owing to the different intrinsic sensitivities of the relevant complexes (monomer $<$ dimer $<$ trimer, etc), can perturb signaling (Figure 1C). The prediction of a direct impact of nucleo-cytoplasmic dilution on the assembly of macromolecular complexes in small and large cells deserves experimental validation.

The results of Neurohr et al. are to be correlated with the known cell size increase during aging [2]. Interestingly, old yeast cells (those having undergone $>16$ divisions) grow larger than $200 \mathrm{fL}$, and are at the frontier beyond which RNA and protein synthesis and cell size are less-than-proportional. Consistently, Neurohr et al. show that young cells grown large and large aged cells share similar phenotypes such as slow cell division, lower density, decreased pheromone sensitivity and global transcriptional changes $[8,9]$ pointing to cytoplasm dilution in large aged cells. Not surprisingly, prolonged $\mathrm{G} 1$ arrest and increased cell volumes decrease lifespan. Interestingly, preventing cell volume increase with cycloheximide or low glucose restores average lifespan.

Increased cell size is also a feature of replicative senescence in human cells ([1] and references therein). Accordingly, primary human fibroblasts treated for one day with doxorubicin, a DNA-damaging agent, undergo an 8-fold volume increase 
some days after treatment, which cannot be fully explained by their tetraploidization (which also increases cell volume) [4]. Similar results were obtained after using a Cdk4/6 inhibitor. Moreover, after a 4-day arrest, only $5 \%$ of cells were able to re-enter cell cycle after removal of the inhibitor. Interestingly, senescence could be blocked by preventing cell size increase in cells cultured in serum-poor medium [4]. Measurement of the cytoplasmic diffusion of nanoparticles [10] showed a lower macromolecular crowding and hence viscosity in large cells, pointing to cytoplasmic dilution, as shown for yeast cells [4].

All in all, this paper shows commonalities between aging yeast and mammalian cells suggesting the existence of a range of DNA-content-tonucleo/cytoplasm volume ratio that ensures an optimal cell function beyond which senescence can occur. 
Figure 1. Nonlinearities and nucleo-cytoplasmic dilution. (A) A promoter (green) with two binding sites for a TF (yellow). Dotted lines represent attractive forces giving rise to cooperative promoter recognition. Assuming a strong degree of cooperativity and that the saturation level of the promoter is proportional to transcription, the transcriptional response (TR) of a promoter with $\mathrm{n}$ binding sites for an activator can be described by the equation:

$$
T R=\frac{[T F]^{n}}{K^{n}+[T F]^{n}}
$$

where $\mathrm{K}$ is a constant [11].

(B) Graph showing the normalized TR as a function of TF concentration for promoter with (n) 2 or 3 binding sites (left). The value of $K$ was arbitrarily chosen so that $50 \%$ of TR is reached at $5 \mathrm{nM}$ of TF. Note that the curve for $n=3$ is steeper than for $n=2$. Ratio showing the fold reduction of TR when the TF is diluted 1.33x (dotted lines) or $2 x$ (full lines; similar to what would happen in a case of heterozygous deletion of a haploinsufficient gene) (right). The fold-reduction of TR is proportional to the power $n$ of the dilution factor when the working concentrations of TF are low. For instance, a $2 x$ dilution of the TF entails an 8-fold drop in the activity of the promoter with $n=3$ (highlighted in gray). As a rule, TFs expressed well below $\mathrm{K}$ are predicted to be more sensitive to dilution than abundant TFs. This sensitivity is promoter-dependent and increases with $\mathrm{n}$. For promoters with a sites for $\mathrm{TF}_{\mathrm{A}}, b$ sites for $\mathrm{TF}_{\mathrm{B}} \ldots . . z$ sites for $\mathrm{TF}_{Z}$, the TR equation becomes:

$$
T R=\frac{\left[T F_{A}\right]^{a}\left[T F_{B}\right]^{b} \ldots\left[T F_{Z}\right]^{z}}{K^{\prime}+\left[T F_{A}\right]^{a}\left[T F_{B}\right]^{b} \ldots\left[T F_{Z}\right]^{z}}
$$

and for low TF concentrations the (maximum) fold-reduction of TR is proportional to the power $a+b+\ldots+z$ of the dilution factor.

(C) Enzymes involved in signal transduction are often dosage sensitive. They are also often involved in opposed reactions such as those interconverting substrates $S$ and $S^{*}$ (where the asterisk represents a post-translational modification). 
In the cartoon, enzymes E1 (dimer) and E2 (monomer) have opposed activities on the substrate such as a kinase and a phosphatase, respectively. According to Eq. 1, nucleo-cytoplasmic dilution is supposed to have a greater impact on the dimer than on the monomer and this would alter the balance between unmodified (S) and modified $\left(\mathrm{S}^{*}\right)$ substrate, potentially leading to defective signaling. Several of such modules are represented in many pathways, such as the MAPK pathway [12]. 


\section{References}

1 Biran, A. et al. (2017) Quantitative identification of senescent cells in aging and disease. Aging Cell 16, 661-671

2 Mortimer, R.K. and Johnston, J.R. (1959) Life span of individual yeast cells. Nature 183, 1751-1752

3 Demidenko, Z.N. and Blagosklonny, M.V. (2008) Growth stimulation leads to cellular senescence when the cell cycle is blocked. Cell Cycle Georget. Tex 7, 33553361

$4 \quad$ Neurohr, G.E. et al. (2019) Excessive Cell Growth Causes Cytoplasm Dilution And Contributes to Senescence. Cell 176, 1083-1097.e18

$5 \quad$ Zhurinsky, J. et al. (2010) A coordinated global control over cellular transcription. Curr. Biol. CB 20, 2010-2015

6 Gasch, A.P. et al. (2000) Genomic expression programs in the response of yeast cells to environmental changes. Mol. Biol. Cell 11, 4241-4257

7 Veitia, R.A. (2010) A generalized model of gene dosage and dominant negative effects in macromolecular complexes. FASEB J. Off. Publ. Fed. Am. Soc. Exp. Biol. 24, 994-1002

$8 \quad \mathrm{Hu}, \mathrm{Z}$. et al. (2014) Nucleosome loss leads to global transcriptional upregulation and genomic instability during yeast aging. Genes Dev. 28, 396-408 9 Neurohr, G.E. et al. (2018) Deregulation of the G1/S-phase transition is the proximal cause of mortality in old yeast mother cells. Genes Dev. 32, 1075-1084 10 Delarue, M. et al. (2018) mTORC1 Controls Phase Separation and the Biophysical Properties of the Cytoplasm by Tuning Crowding. Cell 174, 338-349.e20 11 Bottani, S. and Veitia, R.A. (2017) Hill function-based models of transcriptional switches: impact of specific, nonspecific, functional and nonfunctional binding. Biol. Rev. Camb. Philos. Soc. 92, 953-963

12 Ferrell, J.E. and Ha, S.H. (2014) Ultrasensitivity part I: Michaelian responses and zero-order ultrasensitivity. Trends Biochem. Sci. 39, 496-503 